\title{
The Impact Of Economic And Social Orientation On Trust Within Teams
}

Mayukh Dass, Texas Tech University, USA

Piyush Kumar, University of Pennsylvania, USA

\begin{abstract}
In this paper, we develop a model to examine the impact of an individual's economic and social orientation and the congruence of such orientations across the trustor-trusted dyad on the level of dyadic trust within a team. The model explicitly accounts for higher order dependencies in trust among team members and the potential reciprocity in trust between members of a dyad. The results from our model estimation provide evidence that congruence in economic and social orientation is an important antecedent of dyadic trust. We also find that dyadic trust is characterized by higher order dependencies among team members; however, we do not find that trust is characterized by reciprocity. We also find a wide variation in the polarization in teams based on the levels of trust among their members. Taken together, these findings highlight the need to account for higher order dependencies in the study of trust in teams.
\end{abstract}

Keywords: trust within teams; organizational team analysis; bilinear network model; economic and social orientation; team polarization

\section{INTRODUCTION}

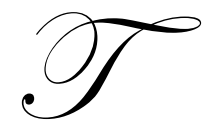

rust is widely acknowledged as an important antecedent of cooperative behavior both within and across organizations because it is one of the forces that bind individuals together in a team as they strive to achieve common goals (Axelrod 1984). It is believed to promote efficient communication and coordination within a team, facilitate the management of slack among team members, focus energy away from issues unrelated to performance, and consequently help improve the quality of collaborative outcomes (Larson and LaFasto 1989). The study of the antecedents and consequences of trust and among members of teams and organizations has therefore remained an important area of research in the social sciences (Gambetta 1988; Good 1988; Mayer, Davis, and Schoorman 1995; McAllister 1995).

One stream of literature on trust focuses on its economic underpinnings and explores the concept within the context of risk-taking in decision-making (Granovetter 1985, Williamson 1993). A central thrust of research within this domain is the examination of how trust influences an actor's subjective beliefs about the probability of future favorable or unfavorable behavior by counter-party in a relationship (Gideon 2007). In a parallel stream of work, however, trust is conceptualized as a social construct that results from long-standing interpersonal relationships (Lewis and Weigart 1985). In a specific set of circumstances, the relative effects of economic and social factors on trust are, of course, expected to be influenced by an individual's own propensity to trust (Good 1988) as well as contextual variables such as the trustor's mood (McAllister 1995).

Taken together, these streams of research suggest that the level of trust across a trustor-trusted dyad may be influenced by both economic and social factors. Economic factors tend to be forward looking and incorporate expectations of likely future behavior by others. Social factor, on the other hand, tend to be anchored in past relationships and are based on the depth of interpersonal bonds between the trustor and the trusted (Jones and George 1998). However, several questions regarding the effects of these two factors remain unaddressed. First, we do not know whether the trustor's economic or social orientation towards the trusted is a stronger driver of dyadic trust. Second, it is unclear whether a high level of overall trust in one direction across a dyad in any way influences the level of trust in the reciprocal direction. 
These questions become more complex when we consider trust in dyadic relationships that are formed within the context of larger teams or organizational systems. In a team environment, the relationship between a trustor and trusted is likely to be influenced by higher order team dynamics and the relationships of the members of a dyad with the remaining team members. In other words, dyadic trust is likely to be influenced by whether other team members trust one or both members of the focal dyad as well as the overall level of polarization in the levels of trust within the team. However, while the relative importance of economic versus social orientation, higher order team dependencies, and the polarization of the team are key issues to the study of dyadic trust within teams, their complete resolution has been constrained, in part, because of an absence of an appropriate methodology.

In this paper, we develop a model to examine the impact of an individual's economic and social orientation and the congruence of such orientations across the trustor-trusted dyad on the level of dyadic trust in a team. The proposed model has several novel features. First, it explicitly accounts for higher order dependencies in trust among team members and then examines their effects on the level of trust at a dyadic level. In other words, it accounts for the influence of the relationships between the trustor and other members of the team on the level of trust on a focal trusted. Specifically, it allows us to examine the common trustor effect, that is, the effect that the levels of trust by a third team member on the individual members of a dyad has on the levels of trust between the two members. It also allows us to examine a common trusted effect, that is, the effect that the levels of trust of the members of a dyad on a third team member has on the level of trust between them. Second, the model incorporates the effects of both individual-specific economic and social orientation as well as the congruence of such orientations between the trustor and the trusted. Therefore, it allows us to examine whether the trustor's own orientation or a match between the orientations of the trustor and the trusted influences the level of trust between them. And, third, the model allows us to examine reciprocity in trust and assess whether the extent to which one team member trusts a second member is in any way related to the extent to which the second team member trusts the first one.

The results from the estimation of our model using data on dyadic trust from executives working in teams provide evidence for the existence of higher order dependencies among the levels of trust in teams. Specifically, we find evidence in support of the common trustor effect which suggests that a higher order dependency exists in the levels of trust between two members who are evaluated similarly by the same external trustor. We also find that both economic and social orientation may be important antecedents of dyadic trust in a team. In our sample, we find that the congruence of economic goals had a greater effect on mutual trust than the congruence of social goals. However, we do not find individual orientation to have significant effect on the levels of trust. Nor do we find trust levels to be characterized by reciprocity in that a higher level of trust by one team member translating into a high level of trust in the reverse direction. Nevertheless, we do find evidence for a wide variation in the polarization of teams based on the levels of trust among its members. While some teams were balanced with similar levels of trust across the members, others were highly polarized with significant imbalances in levels of trust. Taken together, these findings highlight the need to account for higher order dependencies in the study of trust in teams and provide a new methodological approach to address such dependencies.

Our goal in this paper is to make two contributions to the literature on trust within teams. First, we introduce a modeling approach that is capable of examining the effects of social and economic orientation and the congruence therein on dyadic trust in teams after accounting for higher-order dependencies in trust among team members. And second, we develop a mechanism to uncover the extent of polarization in trust within a team based on the level of trust variation among team members.

The rest of the paper proceeds as follows. In the next section, we discuss the relationship between our research and the extant body of literature on trust in teams. Thereafter, we develop our model and discuss its key features. Following that, we describe our study and discuss our key findings pertaining to both the antecedents of dyadic trust and the levels of polarization in teams. We conclude by discussing the theoretical implications of our work and proposing some directions for future research.

\section{RELATIONSHIP WITH THE LITERATURE}

While trust is most frequently defined as the willingness to be vulnerable to the actions of others (Rousseau et al. 1998), multiple definitions of the construct co-exist in the social sciences (Barber 1983; Luhmann 1979; Mayer 
et al. 1995). In the organizational behavior literature in particular, trust has been conceptualized in a several distinct ways. At one end of the spectrum, the emphasis is more on its strategic and calculative dimensions (Burt and Knez 1995). Under this conceptualization, trust is thought of as an element of cooperative behavior that is used to communicate expectations in games where individuals can carry out threats or promises (Lewis and Weigert 1985). At the other end of the spectrum are definitions that highlight the social and ethical factors that underlie trust (Hosmer 1995).

While researchers acknowledge that there in no universally accepted definition of the construct (Rousseau et al.1998) most agree that trust is a multidimensional psychological state (Kramer 1999). It has both cognitive and affective dimensions that may be present to different degrees depending on the contextual characteristics of the situation (McAlister 1995). These two dimensions relate to the economic and social roots of trust. The former captures the expectations of good faith efforts that others will undertake to honor their commitments and not take advantage of the counterparty when the opportunity arises (Hosmer 1995). The latter captures the strength of interpersonal social bonds and represents a collective property of members of a social system (Lewis and Weigart 1985).

\section{Trust in Teams}

While trust is sometimes conceptualized as an individual disposition or a trait, it is generally suggested that it is principally an aspect of a dyadic relationship and may consequently vary within a person across his or her relationships (Schoorman, Mayer, and Davis 2007). Importantly, in many organizational settings, these dyads are themselves embedded inside larger teams or similar social systems. These teams rely on interdependencies among their members in order to achieve individual and team goals (Wilson, Straus, and McEvily 2006). And, when members are not able to fully monitor each other's behavior because of a lack of complete transparency into their actions, they tend to rely on trust in order to ensure an effective functioning of the team (Sheppard and Sherman 1998). An absence of trust can therefore have several adverse consequences, such as a loss of productivity (McAlister 1995), lack of communication (Zucker et al. 1996), and dissatisfaction among team members (Golembiewski and McKonkie 1975).

However, despite the importance of trust in determining the performance and effectiveness of teams (Jones and George 1998), research is scarce on the determinants of trust in groups. This may be not altogether surprising because a team involves several members, and the interdependencies among them make trust within teams a relatively complex phenomenon (Ashleigh and Nandhakumar 2007). While these dependencies increase the importance of trust as an antecedent for effective team functioning, they also increase the methodological challenges to the study of trust in teams. Specifically, in a team setting, it is often not possible to reduce interpersonal trust to either individual trait or even a characteristic of a dyadic relationship. Instead, because several dyads are embedded within a larger group, trust is likely to also depend on group characteristics such as the degree to which the members of a team come together for a common economic purpose (Zand 1972) or the intensity of the social ties that bind them (Weigart 1981). Therefore interpersonal trust in groups is likely to be a function of both the homogeneity of the group and their social connectedness as well as their common objectives and goals. The relative strength of these two antecedents of trust may, of course, depend on the nature and strength of the social relationships as well as the specific economic incentives present in the context (Bonoma 1976).

\section{Economic Orientation}

Real economic actors tend to engage not only in the pursuit of self-interest but also in "opportunism" (Williamson, 1975). The economic underpinnings of trust are therefore tied to its conceptualization as a cognitive process where one makes a rational evaluation of the likely behavior of others in order to assess the risk associated with achieving a self-interest or economic goal. They reflect the confidence of one party or actor to rely on a partner in a purposeful exchange relationship (Moorman, Deshpande, and Zaltman, 1993) and the belief that actions by a partner that will result in positive outcomes (Anderson and Narus, 1990). Economists suggest that some level of trust in such relationships is necessary for pursuing common goals because mere institutional arrangements are often not sufficient to prevent abuse or fraud (Granovetter, 1985). They argue for the role of personal relations and structures in networks that discourage unwarranted behavior. 


\section{Social Orientation}

Relatively recent research suggests that trust also has social underpinnings in addition to economic considerations (Haas and Deseran, 1981). In this stream of work, the construct is conceptualized as a characteristic of social systems that consist of dyads or groups rather than an individual psychological state. It is argued that the need for trust surfaces only when we have interactions or relationships among individuals and that trust therefore rests on an interpersonal or social basis (Lewis and Weigart, 1985). It is built, in part, on the strength of the emotional bonds that result from social interactions among actors and may be motivated in part by the affect for the trusted.

The social bonds that accompany trust ultimately help teams achieve superior performance (Jarvenpaa and Leidner, 1998) and constitute an important source of social capital within people systems. Within an organizational setting in particular, trust helps reduce transaction costs, increase spontaneous sociability among team members, and facilitate appropriate forms of respect to organizational authorities (Kramer, 1999). However, because these social bonds do not form instantaneously, trust is not immediately inherent at the beginning of a social encounter. Instead, it builds up over time as team members are able share feelings and thoughts and build exchange relationships in which they reflect others as trustworthy parties (Jones and George, 1998). Over time, they develop positive affective responses towards other team members that increase the strength emotional bonds among them (Dunn and Schweitzer, 2005, Weber, Malhotra, and Murningham, 2005).

\section{Reciprocity and Higher Order Dependencies}

One of the key issues in the literature on trust is whether the construct exhibits reciprocity. However, the notion of reciprocity can be conceptualized in at least two ways. There can be reciprocity in behavior where a person who is the beneficiary of positive behavior by another subsequently engages in actions that are beneficial to the former (Falk and Fischbacher, 2006, and Song, 2009). On the other hand, there could be reciprocity in the levels of trust itself where a high level of trust by a trustor results in a high level of trust in the reciprocal direction by the trusted. While the second issue remains important, much of the research on trust has been on investigating it as a unidirectional construct with little emphasis on trust reciprocity (Schoorman, Mayer and Davis, 2007).

Further, because dyads are themselves embedded in larger multi-person teams, in addition to reciprocity, we need to also incorporate the effects of other higher order team dynamics in order to understand the antecedents of trust. Specifically, we need to account for transitivity which might result from implicit influences from other team members on a dyad. The presence of transitivity in trusting relationships would mean that if a member $i$ has a high trust level on member $j$ and member $j$ has high trust level on member $k$, then member $i$ should have high trust on member $k$. Such dependencies among group members are the building blocks of the trust equilibrium in a team. In addition, we expect balance to play a role in the level of trust in a team. Balance implies that the trust between member $i$ and member $j$ is based on how the remaining other team members perceive member $j^{l}$.

In summary, we expect that individual economic and social orientation and the congruence of orientations across the dyad will affect the level of interpersonal trust in teams. Trust is also likely to be characterized by reciprocity in that if a trustor has a high level of trust in the trusted then the reciprocal relationship is also likely to be true. And finally, we expect that the level of trust at a dyadic level will be influenced by higher order dependencies across members of the teams. Our proposed model attempts to account for all these factors as potential antecedents of trust.

\section{THE MODEL}

The key construct in our investigation is the level of dyadic trust between one team member (or trustor $i$ ) and another member (or trusted $j$ ) from the same team. The set of these measures of this construct can be represented in the form of a socio-matrix $Y=y_{i j}$ where $y_{i j}$ represents the level of dyadic trust $y$ between trustor $i$ and trusted $j$. Because our goal is to also account for a team member's individual orientation and the impact of team dynamics on

\footnotetext{
${ }^{1}$ Please see Hoff (2005) and Wassermann and Faust (1994) for more details on second order and third order dependencies.
} 
dyadic trust, our model needs to have certain features. First, it needs to account for the effects of individual members' own characteristics that may impact their trust evaluation of other team members. And second, it needs to be flexible enough to control for and estimate higher order group dynamics. Therefore, we considered a bilinear mixed-effects model of trust that incorporates these requirements (Hoff, 2005, and Hoff and Ward, 2004). We later compare this model with a benchmark linear trust model that is traditionally used to model similar data structures (Barrera 2007, and Moorman, Deshpande and Zaltman, 1993).

We begin with a linear model of trust where the effects of dyadic covariates $x_{i j}$ (social and economic congruence) on dyadic trust $y_{i j}$ between trustor $i$ and trusted $j$ can be represented as follows:

$$
y_{i, j}=\beta^{\prime} x_{i, j}+\varepsilon_{i, j}
$$

Because the effects of individual member's traits and higher order group dynamics are captured in the error component of this linear model, the model assumes to have a covariance structure that is exchangeable under identical permutations of the indices $i, j$ of the two members, respectively. In other words, the trust of member $i$ on member $j$ is considered distinct from the trust of member $j$ on member $i$ (i.e. $y_{i, j}$ and $i, j$ are different from $y_{j, i}$ and $j$, $i$ ). Therefore, we represent the joint distribution of $i, j$ 's as a random-effects component of the residual and regard $\varepsilon_{i, j}$ in equation 1 as

$$
\varepsilon_{i, j}=a_{i}+b_{j}+\gamma_{i, j}
$$

where $a_{i}$ represents the effect of individual characteristics of trustor $i, b_{j}$ represents the effect of individual characteristics of trusted $j$ and $\left(a_{i}, b_{j}\right)^{\prime} \sim$ multivariate normal [MVN] $\left(0, \Sigma_{\mathrm{ab}}\right)$.

Therefore,

$$
\left[\begin{array}{l}
a_{i} \\
b_{j}
\end{array}\right] \sim N\left(\left[\begin{array}{l}
0 \\
0
\end{array}\right], \quad\left[\begin{array}{cc}
\sigma_{a}^{2} & \sigma_{a b} \\
\sigma_{b a} & \sigma_{b}^{2}
\end{array}\right]\right)
$$

We also consider $\left(\gamma_{i, j} \quad \gamma_{j, i}\right)^{\prime} \sim \operatorname{MVN}\left(0, \Sigma_{\gamma}\right)$. Therefore,

$$
\left[\begin{array}{l}
\gamma_{i, j} \\
\gamma_{j, i}
\end{array}\right] \sim N\left(\left[\begin{array}{l}
0 \\
0
\end{array}\right], \quad\left[\begin{array}{cc}
\sigma_{\gamma}^{2} & \rho \sigma_{\gamma}^{2} \\
\rho \sigma_{\gamma}^{2} & \sigma_{\gamma}^{2}
\end{array}\right]\right)
$$

Therefore, the covariance structure of the error term is:

$$
\begin{aligned}
& E\left(\varepsilon_{i, j}^{2}\right)=\sigma_{a}^{2}+2 \sigma_{a b}+\sigma_{b}^{2}+\sigma_{\gamma}^{2} \quad E\left(\varepsilon_{i, j} \varepsilon_{j, i}\right)=\rho \sigma_{\gamma}^{2}+2 \sigma_{a b} \\
& E\left(\varepsilon_{i, j} \varepsilon_{k, l}\right)=0 \quad E\left(\varepsilon_{i, j} \varepsilon_{i, k}\right)=\sigma_{a}^{2} \\
& E\left(\varepsilon_{i, j} \varepsilon_{k, j}\right)=\sigma_{b}^{2} \quad E\left(\varepsilon_{i, j} \varepsilon_{k, i}\right)=\sigma_{a b},
\end{aligned}
$$

where $\sigma_{a}^{2}$ represent the variance in the observation due to the trust measured by the same trustor. $\sigma_{b}^{2}$ represents the variance in the observation due to the trust measured by the different trustor on the same trusted, and $\rho$ represents the correlation of observations between a particular pair of members and serves as a measure of reciprocity in group behavior. Thus,

$$
\theta_{i, j}=a_{i}+b_{j}+\gamma_{i, j}
$$


The above equation (3) is modeled as a Gaussian ${ }^{2}$ such that the dyadic data are unconditionally dependent, but conditionally independent because of the given the random effects of the trustor and the trusted members. Therefore,

$$
\begin{aligned}
& E\left(y_{i, j} \mid \theta_{i, j}\right)=g\left(\theta_{i, j}\right), \text { and } \\
& p\left(y_{12}, y_{13}, \ldots y_{n, n-1} \mid \theta_{12}, \theta_{13}, \ldots \theta_{n, n-1}\right)=\prod_{i \neq j} p\left(y_{i, j} \mid \theta_{i, j}\right)
\end{aligned}
$$

\section{Incorporating the Effects of Higher-order Dependence}

Higher order dependencies among members are present and often prominent in most team activities (Wassermann and Faust, 1994). For example, strong dyadic trust between two team members can impact their trust over others. Similarly, strong explicit opinion by a key member about a particular other member, say $A$, can also impact the way other team members perceive $A$.

To capture the effects of such dependencies in our trust model, we construct a latent K-dimensional vector $z_{i}$ and $z_{j}$ for each trustor and trusted respectively and add the inner product $z_{i}^{\prime} z_{j}$ to the error model Equation 2.

Thus,

$\varepsilon_{i, j}=a_{i}+b_{j}+\gamma_{i, j}+z_{i}^{\prime} z_{j}$

Based on the magnitude and direction of the latent vectors, the inner product $z_{i}^{\prime} z_{j}$ captures different higher-order dependence through the expectation of the higher order moment. Further, the incorporation of $z_{i}^{\prime} z_{j}$ into the linear predictor allows for additional moments of $\varepsilon_{\mathrm{i}, \mathrm{j}}$. Therefore,

$$
\begin{aligned}
& E\left(\varepsilon_{i, j}^{2}\right)=\sigma_{a}^{2}+\sigma_{b}^{2}+\sigma_{\gamma}^{2}+K \sigma_{z}^{4} \\
& E\left(\varepsilon_{i, j} \varepsilon_{j, i}\right)=\rho \sigma_{\gamma}^{2}+2 \sigma_{a b}+K \sigma_{z}^{4} \\
& E\left(\varepsilon_{i, j} \varepsilon_{k, l}\right)=K \sigma_{z}^{6}
\end{aligned}
$$

Because the inner-product term $z_{i}^{\prime} z_{j}$ is a fixed effect, it can be considered as a reduced-rank interaction term. This is typically known as the bilinear effect or multiplicative interaction (Hoff, 2005). To include this inner product of the member's latent characteristics in the random effect model, Equation (3) is re-parameterized as:

$$
\begin{aligned}
& \theta_{i, j}=\beta_{d}^{\prime} x_{d, i, j}+\text { tor }_{i}+\text { ted }_{j}+\gamma_{i, j}+z_{i}^{\prime} z_{j} \\
& \text { tor }_{i}=\beta_{t o r}^{\prime} x_{t o r, i}+a_{i} \\
& \text { ted }_{j}=\beta_{\text {ted }}^{\prime} x_{t e d, j}+b_{j}
\end{aligned}
$$

where $x_{d, i, j}$ are dyadic covariates specific to a dyad $i-j$, that is, the level of congruence in the economic and social

\footnotetext{
${ }^{2}$ In our case, a Gaussian model is appropriate, given that we compute the trust level of the trustor $i$ on trusted member $j$ as an average score of four scale items in the questionnaire
} 
orientation of trustor $i$ and trusted $j$ respectively, $x_{t o r, i}$ are the trustor specific covariates, that is, an individual trustor's social and economic orientation, and $x_{\text {ted }, j}$ are the trusted specific covariates, that is, an individual trusted's social and economic orientation.

In other words, the final trust model estimated to determine the effects of economic and social congruencies and individual traits on dyadic trust is as follows:

$$
y_{i, j}=\beta_{d}^{\prime} x_{d, i, j}+\beta_{t o r}^{\prime} x_{t o r, i}+a_{i}+\beta_{t e d}^{\prime} x_{t e d, j}+b_{j}+\gamma_{i, j}+z_{i}^{\prime} z_{j}
$$

This model controls for higher order team dynamics using the bilinear component $z_{i}^{\prime} z_{j}$ and also allows both trustor $\left(x_{t o r, i}\right)$ and trusted $\left(x_{t e d, j}\right)$ characteristics to be investigated along with the dyadic covariates $\left(x_{d, i, j}\right)$.

\section{Data}

We tested our proposed model using data collected from participants in a two-year executive MBA program. These participants had a minimum of five years of work experience and were organized into twenty permanent teams of five members each to work on their course projects for the entire first year of the program. We chose this sample for estimating our model for several reasons. First, all the teams were constituted at the same time and had worked together for the same duration at the time of the study. Second, they were on campus on the same days and had similar opportunities to develop social ties. Third, all teams were part of a lock-step program, were required to undertake similar team-based projects, and had similar team level performance goals. And finally, they were in an environment where there was frequent feedback available on their performance which allowed them to periodically assess how their team was performing on the tasks for which it was constituted.

A survey instrument was developed that contained items on social orientation, economic orientation, and overall trust that were each measured using a 1-7 agree-disagree scale. Each member of a team rated every other member of his or her team on these variables. We measured trust using six items that covered whether a team member thought carefully before sharing information, entered into unwritten contracts, minimized information exchange, checked on each other through third parties, stated obligations clearly, and looked over their shoulder. We measure economic orientation using nine items that covered whether the relationship with the team member helped achieve professional and academic goals. On the other hand, we measured social orientation using eleven items that covered whether the relationship with the team member helped achieve social and friendship related goals. The data were collected midway through the course of the second half of the academic year.

\section{Model Estimation}

We used a Bayesian process to estimate the parameters of the model shown in Equation 5. Specifically, we employed a Markov Chain Monte Carlo (MCMC) algorithm to sample values of the trustor and trusted specific parameters from their posterior distributions. The estimation process constructs a Markov chain in $\left\{\beta_{d}, \beta_{t o r}, \beta_{\text {ted }}, \Sigma_{a b}, Z, \sigma_{z}^{2}, \Sigma_{\gamma}\right\}$ and follows a three-step process of sampling from the desired trusted posterior distribution $p\left(\beta_{\text {tor }}, \beta_{\text {ted }}, \Sigma_{a b}, Z, \rho_{Z}^{2}, \Sigma_{\gamma} \mid Y\right)$. The details of the estimation process are shown in Appendix A. To facilitate the estimation process, we used the 'gbme' function written in R developed by Hoff (2005). We finally used two latent dimensions in our analysis after testing for other number of latent dimensions. ${ }^{3}$

\section{RESULTS}

We ran the Markov Chain Monte Carlo algorithm as shown in Appendix A on our dyadic trust data. Each

\footnotetext{
${ }^{3}$ Five different values of $\mathrm{K}=0$ to $\mathrm{K}=4$ were tested with a four-fold cross-validation procedure as described by Hoff (2005) in his seminal paper. The predictive performances for all the $\mathrm{K}$ values were roughly the same. The biggest improvement in the marginal likelihood criterion was found to be from $K=1$ to $K=2$. Therefore, $K=2$ was selected.
} 
chain was run for 200,000 iterations. Note that our model involves a large number of parameters, including trustor and trusted specific covariates, and terms capturing higher order dependencies. Therefore, following the suggestion of MacEachern and Berliner (1994), we stored every $50^{\text {th }}$ iteration in order to keep the output file to a reasonable size. Outputs from the first 20,000 iterations were considered burn-in and were not recorded.

The posterior mean values of the coefficients and the standard errors of the covariates from the model estimation are reported in Table 1. We find that the level of trust by a trustor on a trusted was significantly influenced by the congruence of both their economic and social orientations ( $\mathrm{p}<0.05$ for both). The coefficient for economic congruency $(0.52)$ is larger than that for social congruency $(0.38)$, suggesting that similar economic goals were perhaps more important among members of our sample than similar social goals. However, we find that the coefficients for the individual orientation of both the trustor and the trusted are not statistically significant ( $p>0.10)$. This suggests that the matching between the orientations of the members of the dyad was more influential in driving trust than either a high or a low level of individual economic or social orientation of the respective members of each dyad.

\section{Impact of Higher Order Dependencies}

Recall that we are also interested in determining the impact of higher order dependencies among team members on the level of dyadic trust between them. The results reported in Table 1 show significant common trustor variance which suggests that higher order dependency in trust existed between members of a dyad who were trusted similarly by a third outside trustor. In other words, if two members were highly trusted by a third member, then the trust between the two members was also high. However, we did not find any significant effect of a common trusted in our study. Importantly, we found the variance of the latent dimensions to be small and similar to that of the errors of the model, suggesting that our model is well calibrated and has captured the higher-order effects of team dynamics on dyadic trust. Finally, the coefficient for reciprocity was positive but not statistically significant $(\mathrm{p}>0.10)$. This is a key finding which shows that a high level of trust by one team member, $i$, on another member, $j$, did not translate into a high level of trust in a reciprocal direction by $j$ on $i$. In other words, only a small degree of within-dyad dependence was present among the team members.

Table 1: Posterior Means and Standard Deviation of Covariates in Bilinear Mixed-effect Trust Model

\begin{tabular}{ll}
\hline \multicolumn{1}{c}{ Covariates } & Coefficients (Std. Dev) \\
\hline Economic Congruency Between Trustor (member $i$ ) and Trusted (member $j)$ & $0.5190^{* *}(0.1569)$ \\
Social Congruency Between Trustor (member $i$ ) and Trusted (member $j)$ & $0.3800^{* *}(0.0180)$ \\
Economic Intent of Trustor (member $i$ ) & $0.0010(0.0046)$ \\
Economic Intent of Trusted (member $j$ ) & $-0.0062(0.0046)$ \\
Social Intent of Trustor (member $i$ ) & $-0.0072(0.0048)$ \\
Social Intent of Trusted (member $j$ ) & $-0.0063(0.0046)$ \\
Reciprocity & $0.0087(0.0193)$ \\
Error Variance & $0.0503^{\dagger}(0.0010)$ \\
Common Trustor Variance & $0.002^{\dagger}(0.0004)$ \\
Common Trusted Variance & $0.00000(0.0001)$ \\
Variance of Latent Dimensions & $0.0554^{\dagger}(0.0159)$ \\
Variance of Inner Dimensions & $0.0624^{\dagger}(0.0167)$ \\
\hline
\end{tabular}

** Significant at $95 \%$ CI

${ }^{\dagger}$ Significantly different from 0 at $95 \%$ CI

\section{Comparison with A Benchmark Model}

We next compare the findings of our bilinear mixed-effects model with a benchmark linear trust model ${ }^{4}$ of

\footnotetext{
${ }^{4}$ We also considered a mixed benchmark model where individual social and economic traits of members are used as covariates along with the congruency measures along with random components to control for individual members average social and economic assessments. Although the directions of effects are found to be similar to the one shown in Table 1, none of them are found to be statistically significant. The results can be obtained from the authors.
} 
the following form.

$y_{p}=\beta_{0}+\beta_{1} \times x_{p_{1}}+\beta_{2} \times x_{p_{2}}+\varepsilon$

In this benchmark model, we consider each dyadic trust (of pair $p$ ) evaluation $y_{p}$ as an independent event and estimate the effect of the economic $\left(x_{p_{1}}\right)$ and social congruencies $\left(x_{p_{2}}\right)$ between the respective team members using a linear model. The results from the estimation of this benchmark linear model are reported in Table 2. Comparing the fit of these two models, we find that the negative log likelihood of the benchmark model is substantially inferior (-241.63) than our proposed bilinear mixed-effects model (-3887.33). The superior fit of our model suggests the presence of higher-order group dynamics in such team activities and the importance of controlling for them during the analysis of dyadic trust. Further, we find that, in the benchmark model, while the coefficients of both the economic congruency (0.063) and social congruency $(0.015)$ are positive, neither one is statistically significant $(\mathrm{p}>0.10)^{5}$. In other words, not only did the benchmark model fit relatively poorly compared to the proposed model, it also failed to appropriately identify some of the key antecedents of dyadic trust.

Table 2: Competing Model Parameter Coefficients

\begin{tabular}{lcc}
\hline & Covariates & Coefficient (Std. Error) \\
\hline Intercept & $4.3412(0.2628)^{* *}$ \\
Economic Congruency & $0.0628(0.0618)$ \\
Social Congruency & $0.0121(0.0672)$ \\
\hline
\end{tabular}

$* *$ Significant at $95 \% \mathrm{CI}$

\section{TEAM POLARIZATION}

Given the empirical evidence on the significant effect of higher-order dependencies on dyadic trust in teams, we next examine the team dynamics based on the social polarization among their respective members. Polarization or the trust variation among team members is one of the most visible outcomes of higher order dependencies. It results from a sense of "us versus them" between subgroups within teams which is typically caused due to imbalance in trust among team members (Munkvold and Zigurs, 2007). Teams can be balanced where the dyadic trust level is equal across all member pairs, or they can be polarized, where there is an inequality in the levels of dyadic trust among the members. The latter situation can potentially lead to conflicts and goal displacements among team members, ultimately resulting in undesirable performance outcomes.

We examine trust imbalance within teams in our sample in two steps. In the first step, we compute each member's location in a latent space characterized by their trust levels in their teams. From this step, we are not only be able to identify different team structures based on the dyadic trusts among the members, but are also be able to identify similar and different teams based on the trust variation among the team members. In the second step, we take the structure of individual teams and micro-analyze their polarization.

In order to estimate the location of each team, we adapt a latent class hierarchical model (Handcock et al., 2007) to evaluate the latent positions of the team members based on their trust assessments. Because we intend to examine team polarization based on level of dyadic trust (high trust and low trust), we recomputed the trust measure as binary with 1 if it was above 4 on the 7-point scale (1-7) and 0 , if it was below $4^{6}$. In this modeling approach, we represent the variation in trust across the members in a latent space, denoted by $Z_{k}$ where $k$ is equal to the number of latent dimensions. The data matrix $\mathrm{Y}=\left[\mathrm{y}_{\mathrm{ij}}\right]$ is viewed as a random variable in a sample space of $Y \subseteq\left\{0, \max \left(y_{i, j}\right)\right\}^{n(n-1)}$ and the probability function to estimate the $z_{i}$ and $z_{j}$ in the latent space is given by:

\footnotetext{
${ }^{5}$ We also performed robustness check for multicollinearity. Both Variance Inflation Factor $(<=1.73)$ and Condition Index $(<=11.54)$ suggest absence of multicollinearity.

${ }^{6}$ Current limitation of the newly developed latent class hierarchical model (Handcock et al. 2007) of only allowing binary network structures as input also contributed to this decision.
} 
$\operatorname{Pr}\left(Y=y \mid \beta_{0}, Z\right)=\prod_{i \neq j} \operatorname{Pr}\left(y_{i, j} \mid \beta, z_{i}, z_{j}\right)$

Using a Poisson likelihood function and two latent dimensions ${ }^{7}$, we estimate the above equation 8 using MCMC routines found in latentnet function (Krivitsky and Handcock, 2008).

\section{RESULTS}

Figure 1 illustrates a plot of different teams in the two-dimensional latent space. The clusters represent the location of the teams, and the extent of dispersion within each cluster represents the polarization in trust among the members of each team. This plot shows two interesting team characteristics of our study sample. First, it demonstrates that the teams differed significantly from one another based on the level of mutual trust among their members. And second, it illustrates three types of polarization structure among teams which we term as a highly polarized environment, where two members are tied at opposite edges of the central member cluster (Team A in Figure 1), semi-polarized environment, where only one member is outside the clusters of other members (Team B in Figure 1) and a balanced environment, where all members are clustered together indicating a non-polarized state (Team $\mathrm{C}$ in Figure 1).

In order to examine each of these three team structures in details, we plot them (Figure 2) using Fruchterman-Reingold (1991) 2-D layout algorithm where the team is represented as a network with the team members as nodes and their connection to one another is based on the level of dyadic trust between them. The first figure (Figure 2a), from Team A, shows a type of highly polarized team where more than one team member is located far from others. In this case, we find that the members of Team A, whom we name Paul, Ringo, and Pete, have similar dyadic trust among them. However, there is a trust imbalance between them and two other members, whom we name John and George with high trust inequality between John and George. Such a structure indicates a state of disequilibrium and a low proportion of reciprocity among team members.

Figure 1: Structural Plots of the Groups

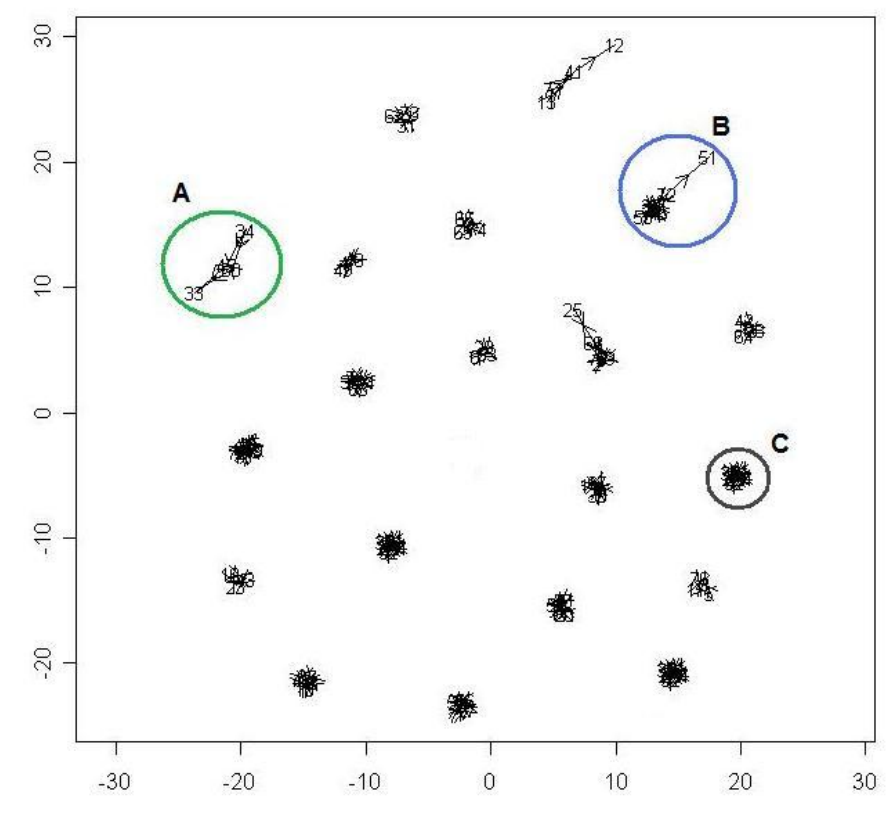

${ }^{7}$ As $k=2$ was used in the previous analysis. See Handcock et al. (2007) for detailed information regarding the estimation of the model. 
Figure 2a: Team-A Highly-Polarized Team

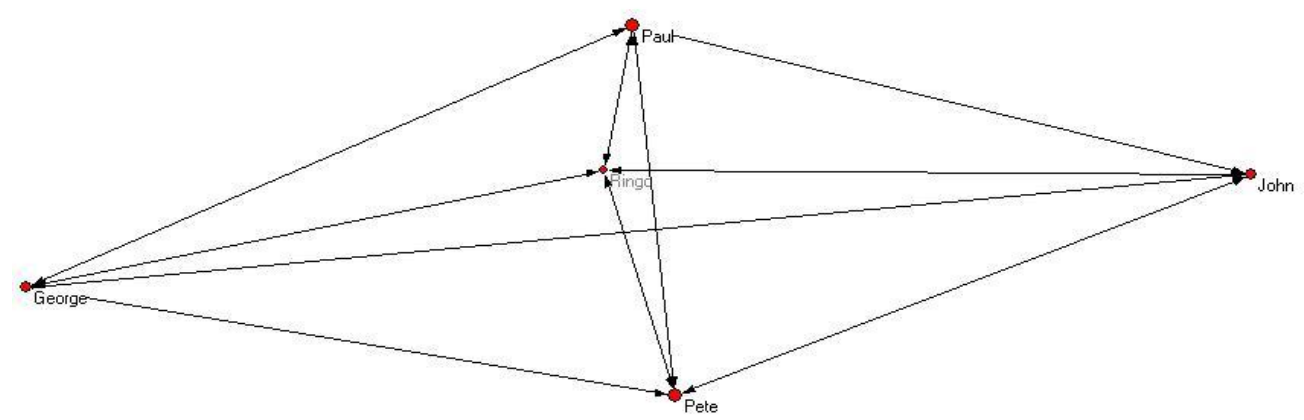

Figure 2b: Team-B Semi-Polarized Team

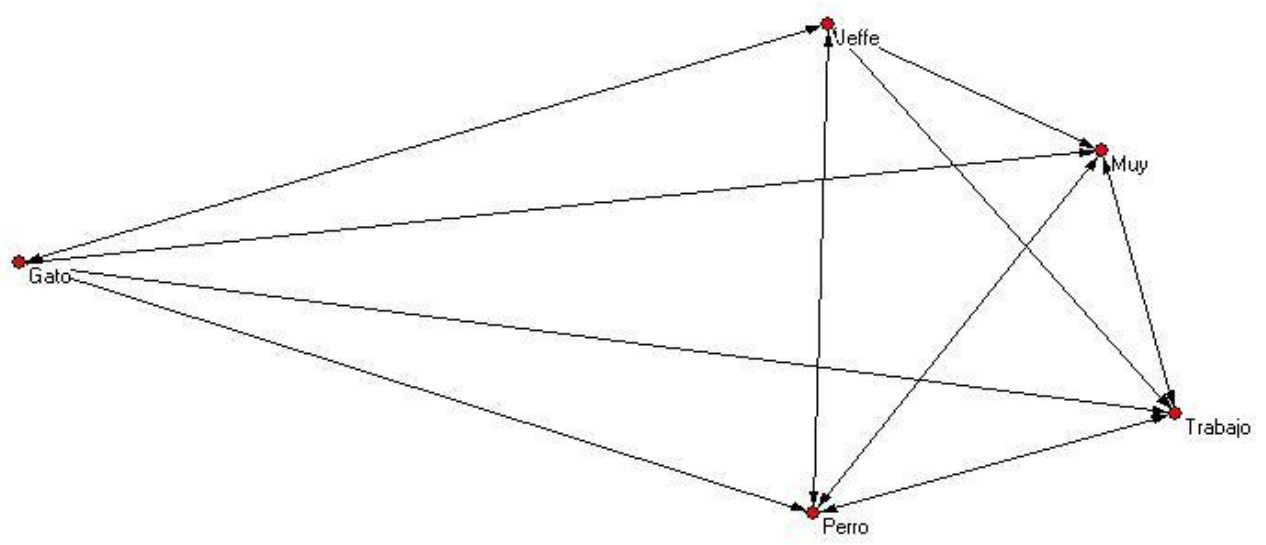

Figure 2c: Team-C Balanced Team

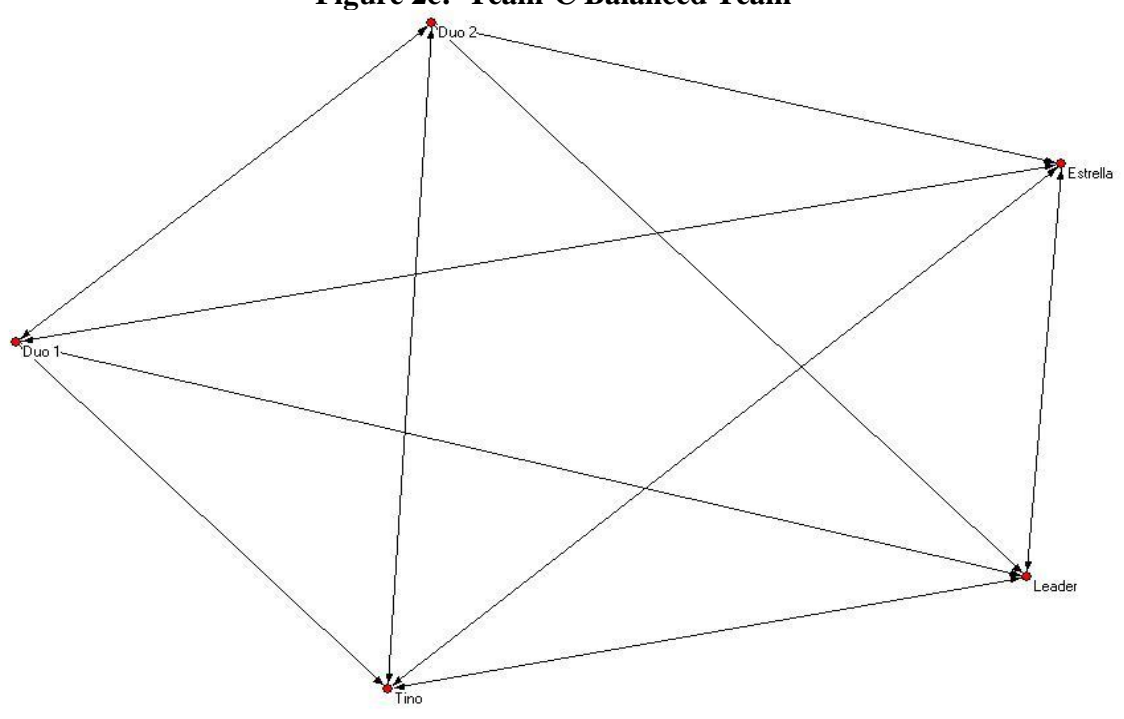


The next team structure (Figure 2b) of Team B illustrates another type of team polarization, which we label as a semi-polarized team. In this case only one team member is in disequilibrium. This member, whom we call Gato, is located far from a closely connected group of members, whom we call Jeffe, Muy, Trabajo, and Perro. This suggests that there is a high variance between the level of Gato's trust of others and others' trust of him. Finally, the third figure (Figure 2c) illustrates a balanced team where every member of the team (Team C) is close to each other, suggesting similar levels of dyadic trust among them. Such a structure further indicates high proportion of reciprocity, strong bond, and greater cooperation among the team members.

\section{DISCUSSION}

Organizations strive to understand the drivers of trust among members of teams in order to build high performance teams. Therefore, it is imperative for them to understand the drivers of trust among team members. However, the examination of interpersonal trust in a team setting is constrained in part because of a lack of appropriate methodologies to investigate this complex phenomenon. In this paper, we develop and estimate a model of dyadic trust in teams in a system consisting of multiple teams. As the scope of our findings suggests, the proposed modeling approach has several advantages over more traditional methods to investigate the antecedents of dyadic trust. It allows us to capture the direct effects of multiple facets of individual orientations such as those pertaining to economic incentives and social relationships. It also allows us to estimate the effects of these individual orientations in a many-to-many context where multi-person teams lead to several, inter-related, bidirectional, dyadic relationships. Our model is able to account for higher order dependencies across team members that are likely to be present in such contexts. Consequently, the results from the estimation of our model do perhaps provide a better explanation for the variation in trust across the system and unearth antecedents of trust that were otherwise found to be not statistically significant using a more traditional linear modeling approach. Finally, our integrated modeling approach also enables us to examine reciprocity of trust in dyadic relationships that is difficult using conventional approaches.

From a substantive point of view, our study shows that, while individual levels of social or economic orientation may not be significant drivers of trust, the congruence of orientations between the trustor and trusted may be. Within our context, we find that the magnitude of the congruence of economic orientation had a stronger effect on trust than the congruence of social orientation. This suggests that it may be relatively less important whether the trustor has high or low social or economic orientation. Trust may be driven more by whether both the trustor and trusted are high or low on either one of the two orientations. In other words, trust is perhaps driven by a match of orientation between the trustor and the trusted rather than necessarily by high or low levels of orientation.

However, we found that dyadic trust was not characterized by reciprocity in that a high level of trust by a trustor on a trusted did not necessarily result in a high level of trust when the roles were reversed. Nevertheless, we found that in our data, higher order dependencies did influence the level of trust between a trustor and trusted. We specifically found evidence in support of the common trustor effect in that a high level of trust by a third member on the actors in a dyad was correlated with a high level of trust within the dyad itself. Overall, our results suggest that a mere congruence of orientation within a trustor-trusted pair may not fully explain the level of trust between them because the latter is influenced by the context created by the fact that the dyad itself is embedded within a larger team.

We do find that the teams were themselves distributed in the latent space and differed in the level of polarization in trust among its members. Within each cluster, while some of the teams were more cohesive and characterized by similar levels of trust, others were less so and demonstrated greater variance in the levels of trust across team members. In other words, the pattern of polarization ranged from balanced to highly polarized. Not only are these patterns informative in and of themselves, they also provide support for our contention that higher order dependencies need to be examined and accounted for in the study of dyadic trust in teams.

Taken together, our study findings suggest that, individual, dyadic, and systemic factors may influence trust between team members in an organizational setting. While individual traits or personality characteristics, such as innate trustworthiness have been found to be important drivers of trust, we find that each of the two orientations considered in our research were themselves not significant drivers of trust. However, the dyadic congruence or 
match in the levels of orientation did affect trust. Further, trust was characterized by higher order dependencies among team members.

While our model and our study contribute to the literature on dyadic trust in teams, the work can be extended in several ways. First, while we focused on economic and social orientation as the key individual level antecedents of trust, the model is flexible enough to accommodate the effects of other covariates and the congruence among them. Second, we chose to conduct our empirical analysis in a setting where there was a high degree of homogeneity among the members of the teams, their overall goals, and the opportunities for them to develop social ties. Future research should calibrate the model with more heterogeneous teams in order to complement our findings regarding the relative impact of the individual orientations, congruence, and higher order dependence. Finally, our study utilized a cross section of data and provides a snapshot of the drivers of trust at a point in time. However, the approach can be extended to longitudinal analyses to improve our understanding of the evolution of trust in teams.

\section{AUTHOR INFORMATION}

Mayukh Dass is an Assistant Professor of Marketing at the Rawls College of Business, Texas Tech University. He holds a Ph.D. in Business Administration, an M.S. in Statistics and an M.S in Artificial Intelligence from the University of Georgia. His research focuses on statistical and analytical methods with applications to valuation issues in dynamic economies and brand management. He is a member of the INFORMS and the Academy of Marketing Science.

Piyush Kumar is visiting associate professor of marketing at the Wharton School of Business, University of Pennsylvania, and associate professor of marketing at the Terry College of Business, University of Georgia. His research interests include web analytics, Internet-based business models, brand management and analytics, and service management. His research has been supported by the Marketing Science Institute, the Institute for the Study of Business Markets, Purdue Research Foundation, and H-E-B. He holds a B.Tech in mechanical engineering from the Indian Institute of Technology, Kanpur, an M.B.A. from the Indian Institute of Management, Ahmedabad, and a $\mathrm{Ph} . \mathrm{D}$. from Purdue University.

\section{REFERENCES}

1. Anderson, J. C. \& Narus, J. A. (1990). A model of distributor firm and manufacturer firm working partnerships. Journal of Marketing, 54, 42-58.

2. Ashleigh, M. J. \& Nandhakumar, J. (2007). Trust and technologies: Implications for organizational work practices. Decision Support Systems, 43, 607-617.

3. Axelrod, R. (1984). The evolution of cooperation. New York: Basic Books.

4. Barber, B. (1983). The logic and limits of trust. New Brunswick, NJ: Rutgers University Press.

5. Barrera, B. (2007). The impact of negotiated exchange on trust and trustworthiness. Social Networks, 29 (2007), 508-526.

6. Bonoma, T. W. (1976). Conflict, cooperation and trust in three power systems. Behavioral Science, 21, 499-514.

7. Burt, R. \& Knez, M. (1995). Kinds of third-party effects on trust. Journal of Rational Sociology, 7, 255292.

8. Dunn, J. R. \& Schweitzer, M. E. (2005). Feeling and believing: The influence of emotion on trust. Journal of Applied Psychology, 88, 736-748.

9. Falk, A. \& Fischbacher, U. (2006). A theory of reciprocity. Games and Economic Behavior, 54, 293-315.

10. Fruchterman, T. M. J. \& Reingold, E. M. (1991). Graph drawing by force-directed placement. Software Practice and Experience, 21(11),1129-64.

11. Gambetta, D. G. (1988). Can we trust trust? In Gambetta, D.G. (Ed.), Trust, New York, NY: Basil Blackwell

12. Gideon, K. (2007). Framing, intentions, and trust-choice incompatibility. Organizational Behavior and Human Decision Processes, 103, 238-255. 
13. Golembiewski, R. \& McKonkie, M. (1975). The centrality of interpersonal trust in group processes. In C.L. Cooper (Ed.), Theories of group processes (pp. 131-185). New York: Wiley.

14. Good, D. (1988). Individuals, interpersonal relations and trust. In Gambetta, D.G. (Ed.), Trust, New York, NY: Basil Blackwell

15. Granovetter, M. (1985). Economic action and social structure: The problem of embeddedness. American Journal of Sociology, 91 (3), 481-510.

16. Haas, D. E. \& Deseran, F. A. (1981). Trust and symbolic exchange. Social Psychology Quarterly, 44, 313.

17. Handcock, M. S., Raftery, A. E. \& Tantrum. J. M. (2007). Model-based clustering for social networks. Journal of Royal Statistical Society - Series A, 170, Part 2, 301-354.

18. Hoff, P. D. (2005). Bilinear mixed effects models for dyadic data. Journal of the American Statistical Association, 100 (469), 286-95.

19. Hoff, P. D. \& Ward, M. D. (2004). Modeling dependencies in international relations networks. Political Analysis, 12 (2), 160-75.

20. Hosmer, L. T. (1995). Trust: The connecting link between organizational theory and philosophical ethics. Academy of Management Review, 20(2), 379-403.

21. Jarvenpaa, S. L. \& Leidner, D. E. (1998). Communication and trust in global virtual teams. Organization Science, 10(6), 791-815.

22. Jones, G. R. \& George, J. M. (1998). The experience and evolution of trust: Implications for cooperation and teamwork. Academy of Management Review, (23), 531-546.

23. Kramer, R. M. (1999). Trust and distrust in organizations: Emerging perspectives, enduring questions. Annual Review of Psychology, 50, 569-598

24. Krivitsky, P. N. \& Handcock, M. S. (2008). Fitting position latent cluster models for social networks with latentnet. Journal of Statistical Software. 24 (5). 1-23.

25. Larson, C. E. \& LaFasto, M. J. (1989). Teamwork: What must go right/ what can go wrong. Thousand Oaks: Sage.

26. Lewis, J. D. \& Weigert, A. (1985). Trust as a social reality. Social Forces, 63(4), 967-985.

27. Luhmann, N. (1979). Trust and power. Chichester: Wiley.

28. MacEachern, S. N. \& Berliner, L. M. (1994). Subsampling the gibbs sampler. American Statistician, 48(3), 188-190.

29. Mayer, R. C., Davis, J. H. \& Schoorman, F. D. (1995). An integrative model of organizational trust. Academy of Management Review, 20(3), 709-734.

30. McAlister, D. J. (1995). Affect and cognition-based trust as foundations for interpersonal cooperation in organizations. Academy of Management Review, 38, 24-59.

31. Moorman, C., Deshpande, R. \& Zaltman, G. (1993). Factors affecting trust in market research relationships. Journal of Marketing, 57(1), 81-101.

32. Munkvold, B. E. \& Zigurs, I. (2007). Process and technology challenges in swift-starting virtual teams. Information and Management, 44 (2007), 287-299.

33. Rousseau, D. M., Sitkin, B. B., Burt, R. S. \& Camerer, C. (1998). Not so different after all: A crossdisciplinary view of trust. Academy of Management Review, 23 (3), 393-404.

34. Schoorman, F. D., Mayer, R.C. \& Davis, J. H. (2007). An integrative model of organizational trust: Past, present and future. Academy of Management Review, 32 (2), 344-354.

35. Sheppard, B. H. \& Sherman, D. M. (1998). The grammars of trust: A model and general implications. Academy of Management Review, 23, 422-437.

36. Song, F. (2009). Intergroup trust and reciprocity in strategic alliances: Effects of group decision-making mechanisms. Organizational Behavior and Human Decision Processes, 108, 164-173.

37. Wasserman, S. \& Faust, K. (1994). Social network analysis: Methods and applications. New York: Cambridge University Press.

38. Weber, J. M., Malhotra, D. \& Murningham, J. K. (2005). Normal acts of irrational trust: Motivated attributions and the trust development process. Research in Organizational Behavior, 26, 75-101.

39. Weigart, A. J. (1981). Sociology of everyday life. Longman.

40. Williamson, O. E. (1975). Markets and hierarchies, analysis and antitrust implications: A study in the economics of internal organization. New York: Free Press. 
41. Williamson, O. E. (1993). Calculativeness, trust, and economic organization. Journal of Law and Economics, XXXVI, 453-486.

42. Wilson, J. M., Straus, S. G. \& McEvily, B. (2006). All in due time: The development of trust in computermediated and face-to-face teams. Organizational Behavior and Human Decision Processes, 99, 16-33.

43. Zand, D. E. (1972). Trust and managerial problem solving. Administrative Science Quarterly, 17, $229-239$.

44. Zucker, L. G., Darby, M. R., Brewer, M. B. \& Peng, Y. (1996). Collaboration structure and informant dilemmas in biotechnology. In R.M. Kramer \& T. R. Tyler (Eds.), Trust in Organizations: Frontiers of Theory and Research (pp. 90-113). Thousand Oaks, CA; Sage. 


\section{APPENDIX A}

The three steps for model estimation, as suggested by Hoff (2005), are as follows:

1. Sampling of linear effects in the model:

(a) Sample $\beta_{d}$, tor, ted $\mid \beta_{\text {tor }}, \beta_{\text {ted, }}, \Sigma_{\mathrm{ab}}, \Sigma_{\gamma}, \mathrm{Z}, \theta$,

(b) Sample $\beta_{\text {tor }}, \beta_{\text {ted }}$ tor, ted, $\Sigma_{a b}, \Sigma_{\gamma}, \mathrm{Z}, \theta$,

(c) Sample $\Sigma_{a b}$, $\Sigma_{\gamma}$ from their full conditionals

2. Sampling of bilinear effects:

(a) For each bidder $\mathrm{i}=1,2 \ldots \mathrm{n}$, sample $z_{i} \mid\left\{z_{j}: j \neq i\right\}, \theta, \beta$, tor, ted, $\Sigma_{z}, \Sigma_{\gamma}$

(b) Sample $\Sigma_{\mathrm{z}}$ from its full conditional distribution.

3. Update $\left\{\theta_{i, j}, \theta_{j, i}\right\}$ using Metropolis-Hastings step:

(a) $\quad$ Propose $\left[\begin{array}{c}\theta_{i, j}^{*} \\ \theta_{j, i}^{*}\end{array}\right] \sim N\left(\left[\begin{array}{c}\beta^{\prime} x_{i, j}+a_{i}+b_{j}+z_{i}^{\prime} z_{j} \\ \beta^{\prime} x_{j, i}+a_{j}+b_{i}+z_{j}^{\prime} z_{i}\end{array}\right], \Sigma_{\gamma}\right)$

(b) Accept $\left[\begin{array}{c}\theta_{i, j}^{*} \\ \theta_{j, i}^{*}\end{array}\right]$ with probability $\frac{p\left(y_{i, j} \mid \theta_{i, j}^{*}\right) p\left(y_{j, i} \mid \theta_{j, i}^{*}\right)}{p\left(y_{i, j} \mid \theta_{i, j}\right) p\left(y_{j, i} \mid \theta_{j, i}\right)} \wedge 1$

The prior distributions of the parameters are taken as:

- $\quad \beta_{d}$, tor, ted, $\beta_{\text {tor }}, \beta_{\text {ted }}, Z$ are considered as multivariate normal.

- $\quad \Sigma_{\mathrm{AB}} \sim$ inverse Wishart Distribution

- $\quad \sigma_{\text {tor }}^{2}, \sigma_{\text {ted }}^{2} \sim$ iid inverse gamma(1,1), $\sigma_{\gamma}^{2}=\sigma_{a}^{2}+\sigma_{b}^{2} / 4, \rho=\left(\sigma_{a}^{2}-\sigma_{b}^{2}\right) /\left(\sigma_{a}^{2}+\sigma_{b}^{2}\right)$ 